\title{
CHANNEL EQUALIZATION FOR CODED SIGNALS IN HOSTILE ENVIRONMENTS
}

\author{
Kristina Georgoulakis, Sergios Theodoridis \\ University of Athens, Department of Informatics \\ TYPA Buildings, Panepistimiopolis 15784 Athens Greece \\ e-mail: kristina@di.uoa.gr, stheodor@di.uoa.gr
}

\begin{abstract}
In this paper the detection of Trellis Coded Modulated signals corrupted by Intersymbol Interference, Co-Channel Interference and nonlinear impairments is treated as a classification task by means of a Clustering Based Sequence Equalizer-Decoder. The receiver performs jointly decoding and equalization of trellis encoded signals. No specific model is required for the channel or for the interference and the noise, and no code knowledge is needed at the receiver. Complexity reduction of the equalizer is obtained through two suboptimal techniques, a) Cluster's grouping and b) the M-Algorithm. The robust performance of the proposed scheme is illustrated by simulations.
\end{abstract}

\section{INTRODUCTION}

Trellis Coded Modulation (TCM) is a combined coding and modulation scheme, which improves the noise immunity of a digital transmission system without increasing the transmitted power or the required bandwidth [5]. Thus, TCM is a very attractive transmission scheme for modern communication systems, where power and bandwidth efficiency is required.

However, these systems suffer from the presence of different impairments, such as Intersymbol Interference (ISI), Co Channel Interference (CCI) and channel nonlinearities [2]. For example, in satellite communications, where TCM is widely used, system performance is reduced due to CCI coming from adjacent beams and adjacent satellites. Furthermore, because of the limited availability of bandwidth, the transmitted signals must be severely bandlimited and this is a source of ISI [2]. In radio mobile communications, the same impairments appear due to frequency reuse (CCI) and multipath propagation (ISI). In addition, nonlinear signal impairments arise from signal companding in telephone transmission or in amplification processes, whenever amplifiers are operated near to the saturation point (for example in satellite communications) [1].

For the suppression of ISI in TCM systems many different approaches have been investigated. Equalization and decoding procedures are performed either sep- arately [3] or jointly [4]. The latter approach allows a substantial performance improvement over the former at the cost of a higher complexity. Furthermore, the task of detecting trellis encoded signals in hostile environments, contaminated by ISI, CCI and nonlinearities, is also of great interest [2].

In [8] the Clustering Based Sequence Equalizer (CBSE) was proposed for reducing CCI and nonlinear impairments in uncoded, ISI impaired data of baseband systems. CBSE treats equalization as a classification task, thus freeing itself from the need of an explicit adoption of specific models both for channel and interferences. CBSE through its classification approach, can accommodate efficiently any kind of channel and impairments [6]. In contrast, the performance of classical MLSE (Maximum Likelihood Sequence Estimation) is degraded in the presence of channel nonlinearitites and / or colored impairments, [6], [8].

In this paper a complex CBSE-Decoder is proposed, capable of treating complex signals. For the suppression of ISI of TCM signals the receiver performs equalization and decoding jointly. The CBSE-decoder maps directly uncoded data to received samples thus, there is no need for code knowledge at the receiver end.

A major disadvantage of sequence equalizers, performing jointly decoding and equalization, is their large complexity [4]. It is demonstrated by simulations that CBSE-decoder can accommodate efficiently ISI, CCI and nonlinearities in TCM signals, at a reduced complexity by means of two suboptimal techniques : clusters grouping and the M-algorithm.

\section{SYSTEM DESCRIPTION}

Figure 1 represents the discrete time model of a system employing TCM and impaired by ISI, CCI and nonlinearities. The TCM encoder maps its $m$-information bits input sequence $X(t)$ to $m+1$ encoded bits $Z(t)$, which are assigned to a symbol $y(t)$ taken from a $2^{m+1}$-ary signal constellation in accordance with the mapping rule of TCM [5]. More specifically, from the $m$ input bits $m^{\prime}$ $\left(X^{c}(t)\right)$ are the input of the convolutional encoder with rate $m^{\prime} /\left(m^{\prime}+1\right)$. The rest $m-m^{\prime}$ bits $\left(X^{u}(t)\right)$ are 
left uncoded. The $m^{\prime}+1$ outputs of the encoder serve for subset selection and the $m-m^{\prime}$ uncoded bits for selecting a point from the subset [1].

The transmitted data symbol $y(t)$ passes through the ISI channel with complex impulse response $h(t)$. If the channel is assumed to exhibit nonlinearities, then this kind of distortion should also be considered in the system design. To model a complex-valued nonlinear communications channel two nonlinear elements are used, placed before and after the FIR filter of ISI (see figure 1). Finally, complex zero - mean noise and CCI are added to the information signal. The real and imaginary parts of complex noise are both white Gaussian noise processes with variance $\sigma^{2}$ and they are mutually independent. Also the noise and information signals are assumed to be uncorrelated. The interfering signal is assumed to be a TCM signal. In the followings, the assumption of one major interferer holds [8].

Assuming the presence of all the above impairments, the signal appearing at the equalizer input is :

$$
g(t)=u(t)+i(t)+w(t)
$$

where $u(t)$ is the output of the second nonlinear element, i.e., $u(t)=f_{2}(r(t))$, with $f_{2}$ being a nonlinear function (figure 1). The signal $r(t)$ is given by:

$$
\begin{gathered}
r(t)=\sum_{i=0}^{L}\left[q_{d}(i) h_{d}(t-i T)-q_{q}(i) h_{q}(t-i T)\right]+ \\
j \sum_{i=0}^{L}\left[q_{d}(i) h_{q}(t-i T)+q_{q}(i) h_{d}(t-i T)\right]
\end{gathered}
$$

where, $q(t)=q_{d}(t)+j q_{q}(t)$ is the complex output of the first nonlinear element, (i.e., $q(t)=f_{1}(y(t))$, $f_{1}$ being also a nonlinear function) and $h(t)=h_{d}(t)+j h_{q}(t)$ is the impulse response of the channel, having length $L+1$. Moreover, in the above equation, $i(t)$ is given by :

$$
\begin{gathered}
i(t)=\sum_{i=0}^{L^{i}}\left[y_{d}^{i}(i) h_{d}^{i}(t-i T)-y_{q}^{i}(i) h_{q}^{i}(t-i T)\right]+ \\
j \sum_{i=0}^{L^{i}}\left[y_{d}^{i}(i) h_{q}^{i}(t-i T)+y_{q}^{i}(i) h_{d}^{i}(t-i T)\right]
\end{gathered}
$$

with $y^{i}(t)$ being the sequence of the co-channel data and $h^{i}(t)$ the co-channel impulse response. The length of the interfering channel is assumed to be $L^{i}+1$.

\section{CBSE-Decoder}

The CBSE-decoder aims at recovering the uncoder transmitted sequence of information bits $X(t)$, based on the corrupted received sequence of coded symbols $g(t)$. This method focuses on the clusters that the received data form. The received data are clustered around specific points, whose number and constellation shape is determined by the coding type, the spread of the channel and the impairments characteristics [8].

Let us first assume that the received samples are corrupted by ISI and complex white noise. We denote by $\mathrm{g}(t)=[g(t), \ldots, g(t-D+1)]^{T}$ the vector of $D$ successive received samples. Note that, $D$ is a user defined parameter, and usually in CBSE implementation $D=2$, [6]. If the span of the channel over the transmitted symbols is $L+1$ and the constraint length of the convolutional encoder is $L_{c}=k * m^{\prime}$ then $C=2^{L_{c}+(L+D) * m}$ clusters are formed in the $D$-dimensional space, [8], where $m$ is the number of TCM input bits. The radius of each cluster is determined by the variance of the noise. The existence of CCI multiplies the number of clusters, [7]. When on top of the previous impairments there is also nonlinear distortion, each of the clusters moves to a new position, depending on the form of the nonlinearities.

Each cluster is represented by a suitably chosen representative, which is the complex noiseless channel response vector in the $D$-dimensional space, i.e., $\mathbf{c}(t)=$ $[c(t), \ldots, c(t-D+1)]^{T}$. Each representative corresponds to a specific sequence of successive outcomes $Z(t)$ of TCM encoder or equivalently to a specific sequence of binary TCM inputs $X(t)$. Specificly, $\mathbf{c}(t) \in\left\{\mathbf{c}_{i}, i=\right.$ $1, \ldots, C\}$, with $\mathbf{c}_{i}$ corresponding to one of the possible values of the sequence: $X^{c}(t), \ldots, X^{c}(t-k-L-D+$ 1), $X^{u}(t), \ldots, X^{u}(t-L-D+1)$.

Due to the interdependence that coding and ISI imposes on successive received data, only specific transitions among different clusters are possible. Thus, CBSE-decoder employs a Viterbi type procedure dictated by the specific transitions among the clusters. The Viterbi procedure constructed for the minimum path search is based on the joint coding and ISI trellis diagram, [4]. In the resulting combined trellis there are $2^{L_{c}+(L+D-1) * m}$ states corresponding to :

$$
\begin{array}{r}
S(t)=\left(X^{c}(t-1), \ldots, X^{c}(t-k-L-D+1),\right. \\
\left.X^{u}(t-1), \ldots, X^{u}(t-L-D+1)\right)
\end{array}
$$

In the Viterbi trellis diagram, the transition from a state $S(t-1)$ to a state $S(t)$ corresponds to the emission of a specific cluster representative, indicated by the sequence of symbols formed by the current state and the new information symbol transmitted. This sequence is called label, and it corresponds to the data: $\xi(t)=$ $\left(X^{c}(t), \ldots, X^{c}(t-k-L-D+1), X^{u}(t), \ldots, X^{u}(t-L-\right.$ $D+1))$.

For the completion of the Viterbi Algorithm procedure an appropriate distance metric should be adopted. A popular distance metric in classification problems is the Mahalanobis distance, defined as

$$
D_{i}=\left(\mathrm{g}(t)-\mathbf{c}_{i}\right)^{H} \Sigma_{i}^{-1}\left(\mathrm{~g}(t)-\mathbf{c}_{i}\right)
$$

where $\Sigma_{i}$ is the covariance matrix of each cluster defined, 
for the general case of complex signals, as :

$$
\Sigma_{i}=E\left[\left(\mathrm{~g}(t)-\mathbf{c}_{i}\right)\left(\mathrm{g}(t)-\mathbf{c}_{i}\right)^{H}\right]
$$

where $H$ is the Hermitian Transpose. The use of non diagonal $\Sigma_{i}$ permits the exploitation of the underlying shape of the clusters in cases where clusters have not spherical distribution (i.e., in the presence of non white interference and / or in the case of clusters grouping, [8]).

From the above description, it is apparent that for the CBSE-decoder operation knowledge of clusters representatives and of the matrix $\Sigma_{i}$ is required (see eq. $5)$. Training of the clusters representatives $\left(\boldsymbol{c}_{i}\right)$ is based on uncoded data and it is achieved during the training period. For example, training of representatives can be achieved by a simple averaging of all the data vectors $g(t)$, belonging to the respective cluster, [6]. The matrix $\Sigma_{i}$ can be similarly estimated and adapted. In the sequel, we have assumed that the covariance matrix is independent on the specific cluster, that is : $\Sigma_{i} \equiv \Sigma, \forall i$.

It should be emphasized here, that, the trellis diagram assumed for the CBSE-decoder construction is based on uncoded data (see eq. 5). Thus, in the training period the formed data clusters can be directly mapped to uncoded data labels. That is, due to the classification nature of the CBSE-decoder, the learning procedure is based on uncoded data and the need for code knowledge in the receiver is surmounted. In contrast, in the Viterbi equalizer-decoder [4] explicit knowledge of the employed code is needed.

\section{COMPLEXITY TECHNIQUES}

REDUCTION

The complexity of a sequence receiver, performing jointly decoding and equalization, grows exponentially with channel memory and the constraint length of the trellis encoder. The CBSE-decoder in its full implementation assumes more states than a conventional Viterbi equalizer due to the higher dimension used in CBSE (number of states : $2^{L c+(L+D-1) * m}, D=1$ for Viterbi equalizer, $D \geq 2$ for CBSE).

In this paper, two suboptimal techniques are adopted. The first method is based on clusters' grouping and in the second method the M-Algorithm is used for complexity reduction, [8].

\section{SIMULATION RESULTS}

In the presented simulations 8-PSK signaling format is assumed. The Trellis Coded Modulation scheme employed assumes $m=2, m^{\prime}=1$ and $L_{c}=2$. The four state convolutional encoder and the signal mapping rules are according to [5]. System performance is investigated through Bit Error Rate (BER) for different Signal to Noise Ratio (SNR) and Signal to Interference Ratio (SIR) values.

ISI impaired signal
Initially, the operation of CBSE-Decoder for TCM signals corrupted by ISI is investigated both in the full and the reduced states version of the equalizer. In the simulations the system of Figure 1 is employed but without the co-channel interferer and with an assumption of linear channel (ignoring nonlinear elements). The complex channel impulse response is: $(0.4313+j 0.4311)\left(1-(0.5+j) z^{-1}\right)\left(1-(0.35+j 0.7) z^{-1}\right)$.

Figure 2 demonstrates the loss in performance versus complexity. From the figure, it becomes apparent that the loss of performance of CBSE-decoder employing clusters grouping (64 or 16 states) is not high, compared to the performance of the optimum CBSE-decoder (256 states). The performance - complexity tradeoff obtained with the use of $\mathrm{M}$-algorithm is even better. However, the clusters grouping method offers the advantage that only the reduced number of clusters centers needs to be determined in the training phase.

\section{ISI and CCI impaired signal}

In the next experiments, $\mathrm{CCI}$ is added to the main signal (the channel remains linear). The interfering channel is assumed to be the: $0.3482+0.8704 z^{-1}+j 0.3482+$ $j 0.8704 z^{-1}$. The SIR $(=8 \mathrm{~dB})$ is chosen low in order to account for the augmented levels of CCI in modern communication systems.

The performance of CBSE-decoder is compared with the performance of the Viterbi equalizer performing jointly decoding and equalization [4]. From figure 3, the superior performance of CBSE against the performance of the Viterbi equalizer, under a low SIR, is verified.

ISI, CCI impairments and nonlinear channel

Finally, experiments considering the full structure presented in figure 1 are performed. The ISI and CCI channel impulse responses are as previous. The first nonlinear element is defined as :

$$
q(t)=\frac{2 y(t)}{1+|y(t)|^{2}} \exp \left(\frac{j \pi}{3} \frac{|y(t)|^{2}}{1+|y(t)|^{2}}\right)
$$

This kind of nonlinearity causes an amplitude and phase distortion of the signal and is used to represent the nonlinear high power amplifier in the transmitter [9]. The second nonlinear element is a third order complex Volterra nonlinearity :

$$
u(t)=r(t)+0.1 r^{2}(t)-0.1 r^{3}(t)
$$

The performance of CBSE-decoder and MLSE ([4]) is presented in figure 4. The MLSE equalizer uses a simple linear channel estimation with RLS. The main and interfering channels are those presented in (10) and (11) and SIR=10dB. From the figure the superior performance of CBSE-decoder compared to that of MLSE is verified.

The above results have also been verified for a number of different signal settings (i.e. type of coding, modulation) and for different impairments (channel, interferences). All the results indicate that CBSE-decoder is an 
effective and robust receiver for coded signals corrupted by varying impairments. The classification properties of the equalizer are utilized to perform jointly decoding and equalization without code knowledge and without restrictive channel assumptions. Moreover, the procedure is the same independent of the presence or not of nonlinearities. Furthermore, the sequential nature of CBSE decision mechanism, ensures its high performance.

\section{References}

[1] E.Biglieri, D.Divsalar, P.J.McLane, M.K.Simon Introduction to Trellis Coded Modulation with applications, N.Y., Macmillan Publ., 1991.

[2] I.K.Hwang, L.Kuzz, "Digital Data Transmission over Nonlinear Satellite Channels," IEEE Trans. on Communications, vol. 41, pp. 1694-1702, Nov. 1993.

[3] K.Zhou, J.Proakis, F.Ling, "Decision Feedback Equalization of Time Dispersive Channels with Coded Modulation," IEEE Trans. on Communications, vol. 38, pp. 18-24, Jan. 1990.

[4] P.R.Chevillat, E.Eleftheriou, "Decoding of Trellis Encoded Signals in the presence of Intersymbol Interference and Noise," IEEE Trans. on Communication, vol. 37, pp.669-676, Jul. 1989.

[5] G.Ungerboeck, "Channel Coding with Multilevel Phase Signals," IEEE Trans. on Information Theory, vol. IT-28, pp. 55-67, Jan. 1982.

[6] S.Theodoridis, C.F.N.Cowan, C.P.Callender and C.M.S.Lee, "Schemes for equalization of Communications channels with nonlinear Impairments," IEE Proceedings Communications, vol. 142, pp.165-171, June 1995.

[7] S.Chen and B.Mulgrew, "Overcoming CCI using an adaptive RBF equalizer," IEEE Trans. on Signal Processing vol. 28, pp. 91-107, 1992.

[8] K.Georgoulakis, S.Theodoridis, "Efficient clustering techniques for channel equalization in hostile environments," Signal Processing, vol. 58, pp. 153164, 1997.

[9] A.Saleh, "Frequency-independent and frequencydependent nonlinear models of TWT amplifiers," IEEE Trans. on Communications, vol. COM-29, Nov. 1981.

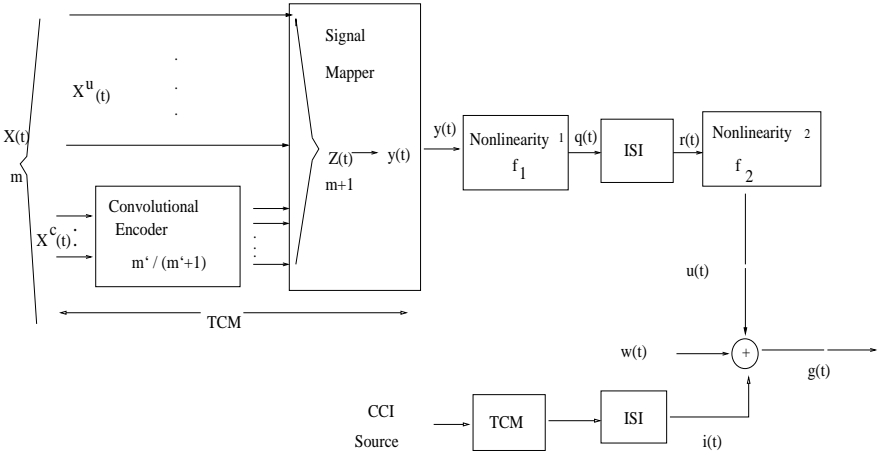

Figure 1: TCM Data transmission system - impaired by ISI, CCI and nonlinearities

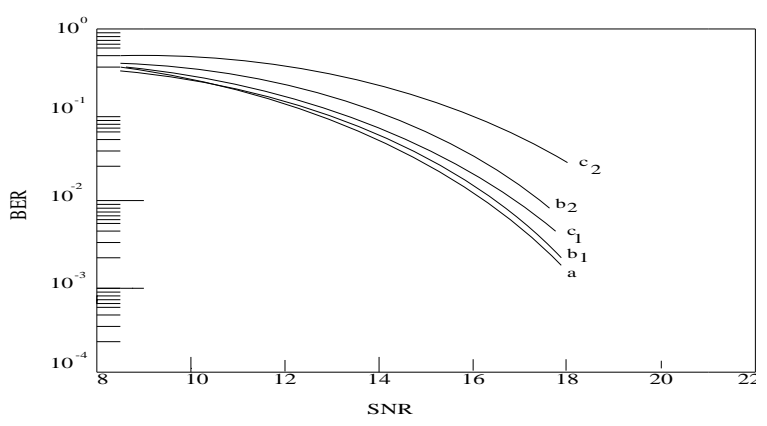

Figure 2: Performance degradation versus complexity reduction, (a) : $\mathrm{Lc}=2, \mathrm{~L}=2-256$ states, M-algorithm : (b1) - 64 states, (b2) - 16 states, Clusters grouping : (c1) - 64 states, (c2) - 16 states

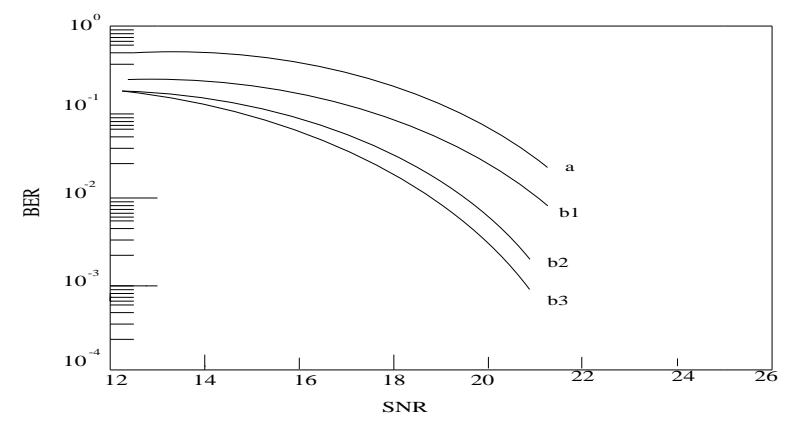

Figure 3: Performance comparison, $\mathrm{SIR}=8 \mathrm{~dB}$, (a) Viterbi Equalizer - 64 states, (b3) CBSE - 256 states, (b2) CBSE - M-algorithm 64 states, (b1) CBSE - Malgorithm 16 states

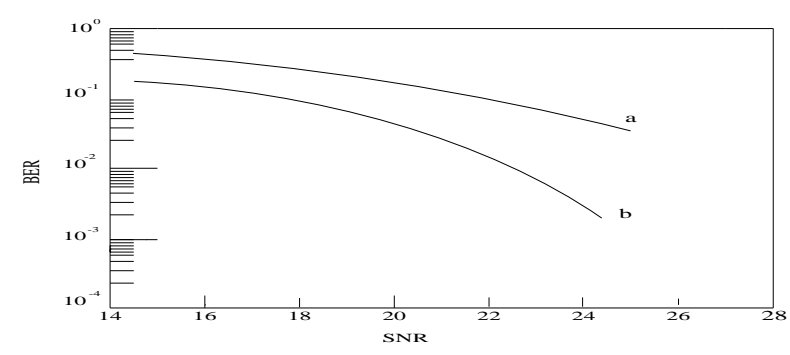

Figure 4: Performance comparison, SIR=10dB, and nonlinear impairments (a) Viterbi Equalizer (b) CBSE 